\title{
Influencia del estado de envejecimiento en el comportamiento mecánico de una aleación de aluminio AA2009 reforzada con partículas de $\mathrm{SiC}^{(\cdot)}$
}

\author{
P. Rodrigo*, P. Poza*, M.V. Utrilla* y A. Ureña*
}

\begin{abstract}
Resumen Se ha realizado un estudio del comportamiento mecánico de un material compuesto de matriz de aleación de aluminio AA2009, reforzada con un $15 \%$ en volumen de partículas de $\mathrm{SiC}$. En dicho estudio se ha evaluado, también, la cinética de endurecimiento aplicando al material un tratamiento térmico T6 a dos temperaturas de envejecimiento distintas (170 y $190^{\circ} \mathrm{C}$ ). El estudio permitió establecer los picos máximos de dureza para las dos secuencias de precipitación. También se evaluaron las propiedades mecánicas a tracción del material compuesto, tratado y sin tratar. El estudio se ha completado analizando los mecanismos de rotura mediante el análisis fractográfico de las probetas ensayadas a tracción y el estudio de las secciones longitudinales utilizando microscopía electrónica de barrido (SEM). Así mismo, se han estudiado, mediante microscopía electrónica de transmisión (TEM), los materiales tratados para determinar el efecto del refuerzo en la distribución de fases endurecedoras precipitadas en la matriz.
\end{abstract}

Palabras clave Material compuesto. Matriz de aluminio. Cinética de envejecimiento. Propiedades mecánicas.

\section{Effect of ageing on the mechanical behaviour of alluminium alloy AA2009 reinforced with SIC particles}

\begin{abstract}
A study of the mechanical behaviour of an aluminium matrix composite (AA2009) reinforced with 15 volume percent of $\mathrm{SiC}$ particles has been carried out. The ageing kinetic for this material has been evaluated at two different ageing temperatures $\left(170\right.$ and $\left.190^{\circ} \mathrm{C}\right)$. The hardness peaks for the two different precipitation sequences existing in the matrix alloy have been identified. The mechanical behaviour of the composite was also evaluated for the different thermal conditions (as-received and aged). This research has been completed with the identification of the fracture mechanisms by means of observation with scanning electron microscopy (SEM) both of the fracture surface and transversal sections of them. In addittion, transmission electron microscopy (TEM) of the treated composites has been used to determine the influence of the $\mathrm{SiC}$ particles on the distribution of strengthening phases precipitated in the matrix.
\end{abstract}

Keywords Composite. Aluminium matrix. Ageing kinetic. Mechanical properties.

\section{INTRODUCCIÓN}

Los materiales compuestos de matriz de aluminio (MCMAl) reforzados con partículas cerámicas presentan importantes ventajas frente a las correspondientes aleaciones sin reforzar ${ }^{[1]}$. Entre las propiedades mecánicas mejoradas por el refuerzo cerámico cabe destacar el módulo elástico, la resistencia al desgaste y el límite elástico, especialmen- te a alta temperatura. Por estos motivos, es importante conocer la influencia del refuerzo sobre la respuesta de la aleación frente a distintas condiciones de tratamiento térmico. Así mismo, el nivel de endurecimiento alcanzado en los materiales compuestos no depende solamente de la aleación, sino que está condicionado, en gran medida, por la forma, tamaño y distribución de las partículas en la matriz $z^{[2]}$.

(.) Trabajo recibido el día 21 de enero de 2005 y aceptado en su forma final el día 27 de abril de 2005 .

(*) Departamento. de Ciencia e Ingeniería de Materiales. Escuela Técnica Superior de CC. Experimentales y Tecnología. Universidad Rey Juan Carlos, c/ Tulipán s/n, 28933 Móstoles, Madrid, España. alejandro.urena@urjc.es. 
Este tipo de materiales responde a los tratamientos térmicos de forma similar a la aleación matriz pero, en general, su cinética de precipitación es más rápida y las durezas máximas alcanzadas son siempre mayores que las de aleaciones sin reforzar, cuyo comportamiento a fractura y su respuesta al endurecimiento por envejecimiento sigue siendo motivo de investigaciones ${ }^{[3 \text { y } 4]}$. Esta diferencia de comportamiento se debe fundamentalmente a la variación que se produce en la densidad de vacantes y de dislocaciones como consecuencia de la presencia del refuerzo ${ }^{[5]}$. El número de vacantes disminuye en la matriz, tanto más cuanto mayor es el porcentaje del refuerzo, ya que las intercaras matriz/refuerzo y los propios límites de grano, junto a las dislocaciones, actúan como sumidero de defectos puntuales ${ }^{[6]}$. Por el contrario, la densidad de dislocaciones aumenta, especialmente, en la vecindad de los refuerzos, debido a tensiones de origen térmico que aparecen en los MCMAl al enfriarlos desde la temperatura de procesado y como consecuencia de la diferencia entre los coeficientes de expansión térmica de la matriz metálica y el refuerzo cerámico. Estas tensiones se relajan emitiendo dislocaciones desde la intercara matriz/refuerzo al interior de la matriz.

Las aleaciones $\mathrm{Al}-\mathrm{Cu}-\mathrm{Mg}$ sin reforzar muestran dos secuencias de envejecimiento diferentes, dependiendo de la relación $\mathrm{Cu} / \mathrm{Mg}$ y, en estado de sobreenvejecimiento, puede presentar dos fases estables: $\theta\left(\mathrm{Al}_{2} \mathrm{Cu}\right)$ y $\mathrm{S}\left(\mathrm{Al}_{2} \mathrm{CuMg}\right)$. Estas dos transiciones podrían darse, por tanto, simultánea o separadamente ${ }^{[7]}$ :

$$
\begin{aligned}
& \mathrm{SSS} \Rightarrow \text { Zonas } \mathrm{GP} \Rightarrow \theta^{\prime \prime} \Rightarrow \theta^{\prime} \Rightarrow \theta\left(\mathrm{Al}_{2} \mathrm{Cu}\right) \\
& \mathrm{SSS} \Rightarrow \text { Zonas } \mathrm{GPB} \Rightarrow \mathrm{S}^{\prime \prime} \Rightarrow \mathrm{S}^{\prime} \Rightarrow \mathrm{S}\left(\mathrm{Al}_{2} \mathrm{CuMg}\right)
\end{aligned}
$$

Una de las principales limitaciones de los MCMAl es su baja ductilidad y tenacidad de fractura. Al aumentar el porcentaje de refuerzo, disminuye la capacidad de alargamiento del material. La rotura de estos materiales tiene lugar por un proceso de nucleación, crecimiento y coalescencia de hue$\cos ^{[8]}$. La nucleación de huecos suele producirse por rotura frágil del refuerzo cerámico o por descohesión de la intercara matriz-refuerzo. Este último mecanismo es el que se ha observado, habitualmente, en materiales reforzados con whiskers o partículas de pequeño tamaño ${ }^{[9]}$. La descohesión del refuerzo cerámico está influida por la precipitación de la matriz. La aparición de fases estables en la intercara favorece la nucleación de huecos en las aristas del refuerzo. Una buena unión interfacial es importante para retrasar, en la medida de lo posible, el fallo del material, puesto que, la etapa controlante en el mecanismo de fractura es la nucleación de defectos, mientras que el crecimiento y la coalescencia de huecos no requieren una gran energía interfacial ${ }^{[1]}$.

En el presente artículo se estudia la influencia de las condiciones de maduración (temperatura y tiempo) en el comportamiento a tracción de una aleación de aluminio basada en el sistema $\mathrm{Al}-\mathrm{Cu}-\mathrm{Mg}$, designada por la Aluminium Association (ASISSAE) como AA2009, reforzada con partículas de $\mathrm{SiC}$. Esta aleación ha sido diseñada para ser reforzada de forma discontinua por su excelente combinación de resistencia y tolerancia al daño. Una característica del refuerzo empleado es su reducido tamaño que, como han demostrado otros autores $^{[10]}$, influye de forma decisiva en la cinética de endurecimiento debido a la mayor incorporación de intercaras $\mathrm{SiCp} /$ matriz en el material. El estudio se centra, por tanto, en determinar para el tipo de aleación y naturaleza del refuerzo empleados, las condiciones de endurecimiento máximo, identificando el estado de precipitación en dicha condición y los mecanismos de fractura dominantes en este estado, bajo condiciones de carga uniaxial.

\section{PROCEDIMIENTO EXPERIMENTAL}

El material compuesto, objeto de estudio, consiste en una aleación de aluminio, AA2009, reforzada con un $15 \%$ en volumen de partículas de $\mathrm{SiC}$ ( $\mathrm{SiCp})$. Es un material comercial fabricado por ACMC (Advanced Composites Materials Corporation) por vía pulvimetalúrgica, recibido en forma de chapones extruidos de $13 \mathrm{~mm}$ de espesor y que no ha recibido tratamiento térmico posterior a la extrusión. La aleación de aluminio AA2009, que ha sido específicamente desarrollada para la fabricación de materiales compuestos de matriz de aluminio de refuerzo discontinuo, está compuesta, básicamente, por $\mathrm{Al}-\mathrm{Cu}-\mathrm{Mg}$ y su composición nominal se muestra en la tabla I.

El material base se sometió a un tratamiento térmico de envejecimiento artificial (T6), consistente en una solubilización del material a $504 \pm$ $1{ }^{\circ} \mathrm{C}$ durante $4 \mathrm{~h}$, seguida de un temple en agua/hielo $\left(0^{\circ} \mathrm{C}\right)$. El envejecimiento, llevado a cabo en un baño de aceite de silicona, se realizó a dos temperaturas diferentes: 170 y $190 \pm 1{ }^{\circ} \mathrm{C}$, durante tiempos comprendidos entre $15 \mathrm{~min}$ y $33 \mathrm{~h}$. La respuesta del material al tratamiento térmico se evaluó mediante ensayos de dureza y microdureza Vickers, siendo el valor obtenido para cada estado 
Relación entre los mecanismos de alimentación y el modo de solidificación en una aleación de aluminio 380 con distintos contenidos de hierro D.O. TOVIO, A.C. GONZÁlEZ, G.W. MúgICA y J.C. CuYás

Tabla I. Composición nominal de la aleación AA2009 (\% en peso)

Table I. Nominal composition of the matrix alloy (weight \%)

\begin{tabular}{cccccccccc}
\hline Aleación & $\mathrm{Cu}$ & $\mathrm{Mg}$ & $\mathrm{Zn}$ & $\mathrm{Si}$ & $\mathrm{Fe}$ & $\mathrm{O}$ & Otros (cada uno) & Otros (total) & Al \\
\hline AA2009 & $3,2-4,4$ & $1,0-1,6$ & 0,10 & 0,25 & 0,07 & 0,6 & 0,05 & 0,15 & resto \\
\hline
\end{tabular}

una media de 10 medidas. Al tener el refuerzo dimensiones muy pequeñas (en torno a $2-3 \mu \mathrm{m}$ ) y una distribución bastante homogénea, las huellas de dureza, realizadas con una carga de $50 \mathrm{~N}$ durante un tiempo de $15 \mathrm{~s}$, incidían sobre matriz y refuerzo conjuntamente. Las microdurezas se aplicaron con $0,5 \mathrm{~N}$ de carga durante el mismo tiempo y procurando que incidieran sólo sobre la matriz.

Para determinar la secuencia de precipitación se realizaron ensayos de Calorimetría Diferencial de Barrido (DSC) sobre el material solubilizado y templado. El ensayo registró la diferencia en el flujo de calor entre la muestra y la referencia, desde 25 a $500{ }^{\circ} \mathrm{C}$, con dos velocidades de calentamiento distintas, 10 y $30^{\circ} \mathrm{C} /$ minuto.

Una vez identificados los picos de envejecimiento se realizaron ensayos de tracción en la dirección paralela a la extrusión, tanto del material base como del tratado. Las probetas de tracción empleadas se fabricaron según las recomendaciones de la norma UNE-EN $10002-1^{[11]}$ para probetas de tracción cilíndricas proporcionales. Los resultados de los ensayos se presentaron en forma de tensión verdadera $[\sigma \mathrm{t}=\sigma(1+\varepsilon)]$ frente a deformación verdadera $[\varepsilon t=\ln (1+\varepsilon)]$ para comprobar el efecto del endurecimiento por deformación de la matriz en los diferentes estados de envejecimiento ensayados.

La microestructura de los materiales en estado de recepción y después de los tratamientos térmicos se estudió mediante microscopía electrónica de barrido (SEM) y de transmisión (TEM), así como microanálisis de rayos $\mathrm{X}$ (EDX), en muestras preparadas según se ha expuesto en trabajos previos $^{[12]}$. Finalmente, los mecanismos de rotura dominantes se evaluaron por el análisis fractográfico y el estudio de las secciones longitudinales mediante SEM de las muestras ensayadas.

\section{RESULTADOS Y DISCUSIÓN}

\subsection{Microestructura del material en estado de recepción}

La microestructura del material de partida se muestra en la figura 1; en esta composición tridimensional se puede observar el aspecto que ofrece

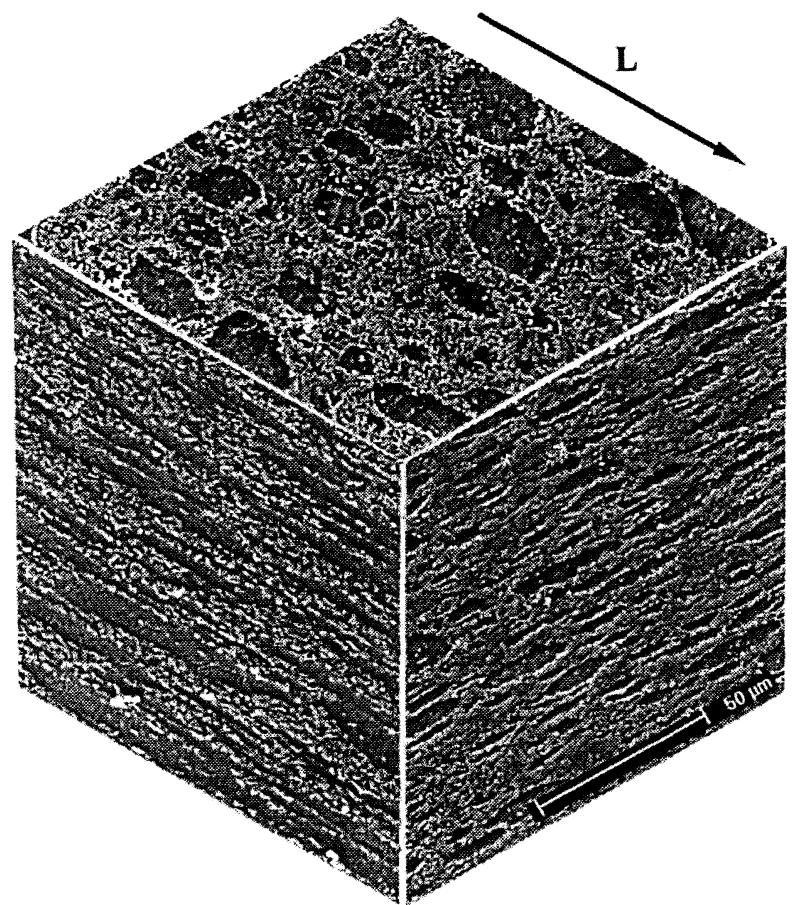

Figura 1. Composición tridimensional de la microestructura del material de partida realizada mediante SEM.

Figure 1. Tree dimensional SEM microstructure of the composite plate.

el material compuesto en función de la dirección de extrusión. El refuerzo de SiCp se caracteriza por su tamaño reducido y uniforme, oscilando entre 2 y $3 \mu \mathrm{m}$ de diámetro medio, y una relación de forma ( $1 / d)$ comprendida entre 2 y 4 . El refuerzo aparece homogéneamente distribuido en la matriz, presentando las irregularidades propias de los materiales compuestos extruidos, es decir, presencia de bandas de mayor y menor densidad de SiCp y agrupaciones locales de refuerzo (clusters); esto hace que existan diferencias microestructurales en las tres secciones ortogonales del material. Se ha comprobado que son las partículas más grandes y alargadas las que presentan una mayor tendencia a acumularse localmente.

Aparecen numerosos precipitados en el material debido a que no fue sometido a ningún tratamiento térmico de solubilización posterior al conformado (Fig. 2a). Se pueden distinguir dos tipos: los de mayor tamaño, en su mayoría asociados a 
zonas de alta densidad de partículas, identificados mediante microanálisis EDX (Fig.. 2b) como la fase $\theta\left(\mathrm{Al}_{2} \mathrm{Cu}\right)$ y, otros más pequeños, y en menor proporción, en cuya composición también se detectó $\mathrm{Mg}$ (Fig. 2c), por lo que fueron identificados como $\mathrm{Al}_{2} \mathrm{CuMg}$ (fase $\mathrm{S}$ ).
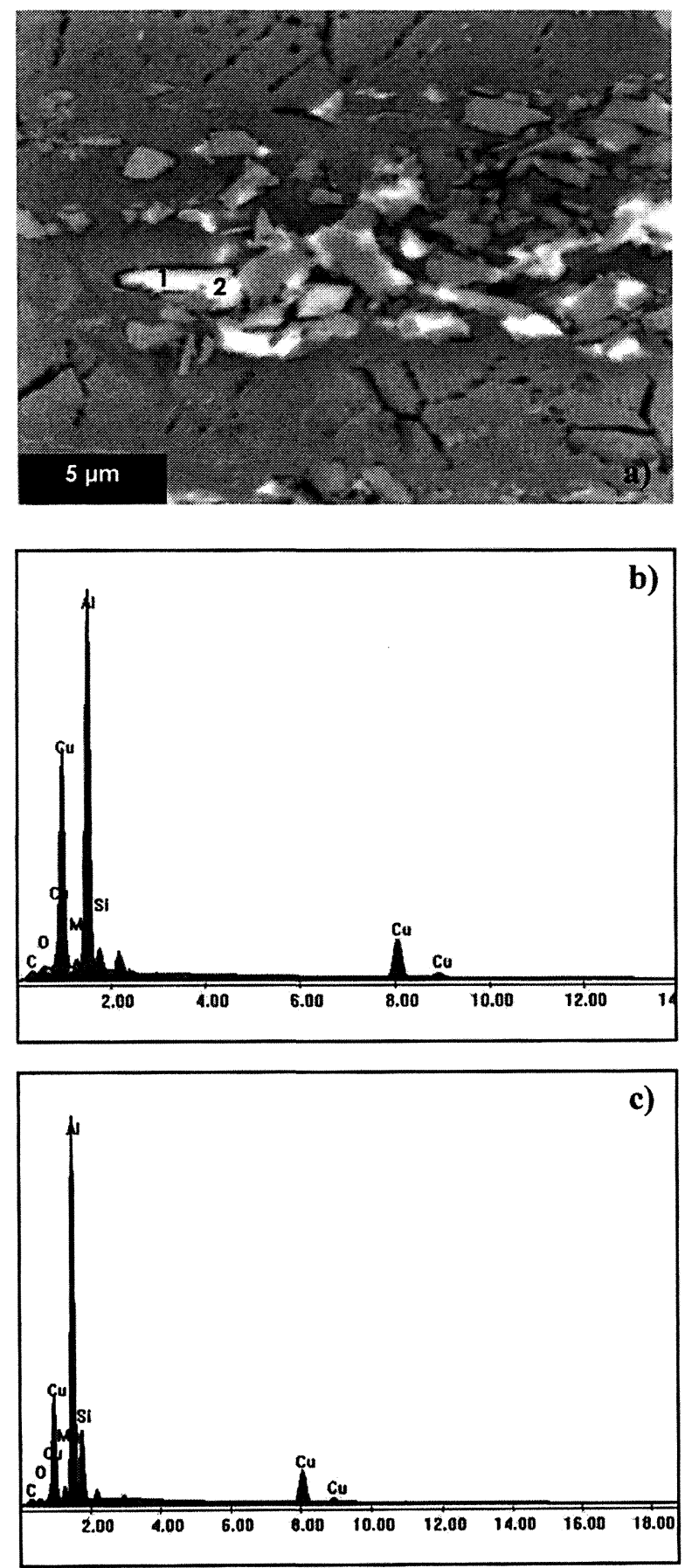

Figura 2. a) Imagen SEM de electrones retrodispersados de una zona de acumulación de partículas. b) Análisis EDX realizado sobre el precipitado marcado con 1. c) Análisis EDX realizado sobre el precipitado marcado con 2 .

Figure 2. a) BSE image of particulate clustering zone. b) EDX microanalysis of the precipitate marked 1. c) EDX microanalysis of the precipitate marked 2.
Se utilizó la microscopía electrónica de transmisión para la completa identificación de estas fases. En la figura 3 se observa un cluster de partículas entre las que se aprecian precipitados de $\mathrm{Al}_{2} \mathrm{Cu}$, identificados por el diagrama de difracción de electrones. Esta imagen muestra también como las intercaras $\mathrm{Al} / \mathrm{SiC}$ se encuentran preferentemente libre de productos de reacción, aunque, excepcionalmente, se han encontrado en ellas algunos óxidos de aluminio o de magnesio, como es normal en materiales fabricados por vía pulvimetalúrgica.

\subsection{Cinética de precipitación}

La variación de dureza y microdureza Vickers en el intervalo de tiempo estudiado para las dos temperaturas de envejecimiento ensayadas $\left(170\right.$ y $\left.190^{\circ} \mathrm{C}\right)$ se muestra en la figura 4. Ambas magnitudes, muestran perfiles de endurecimiento similares entre sí, comprobándose que, independientemente de las variables utilizadas en el tratamiento térmico de envejecimiento, las curvas presentan dos picos de endurecimiento. El primero de ellos, a tiempos de maduración más cortos, se caracteriza por

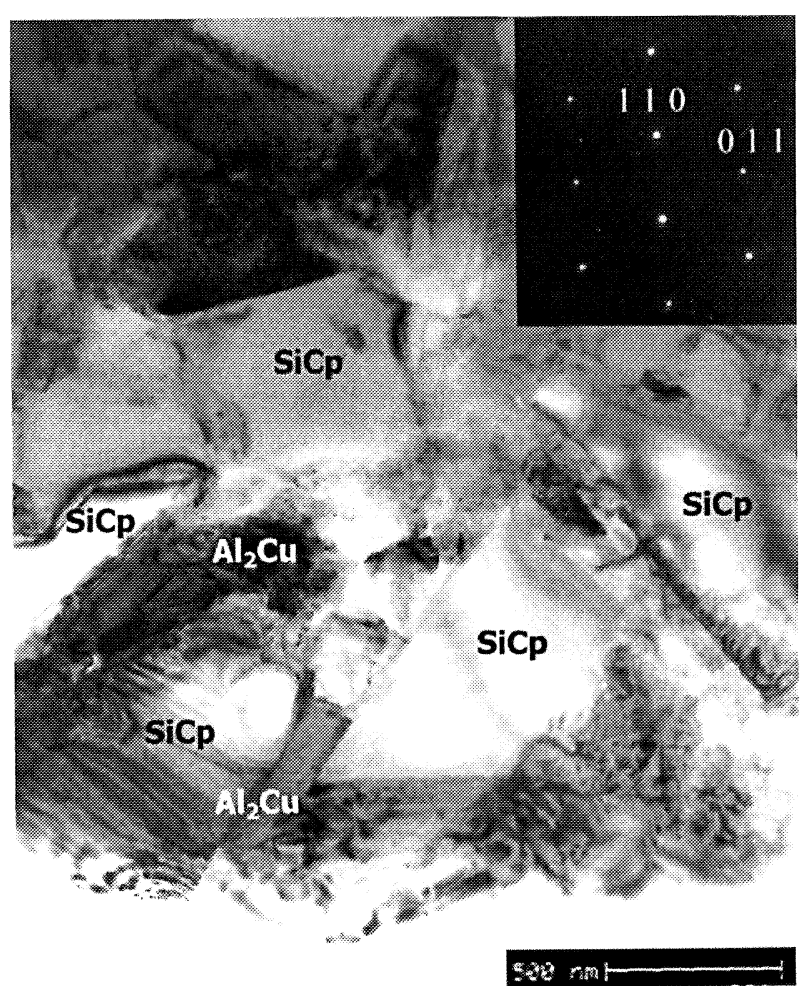

Figura 3. a) Zona de acumulación de partículas entre las que se aprecian precipitados de $\mathrm{Al}_{2} \mathrm{Cu}$, identificados mediante difracción de electrones según eje de zona [ $\left[\begin{array}{lll}1 & 1 & 1\end{array}\right]$.

Figure 3. a) TEM image of the $\mathrm{Al}_{2} \mathrm{Cu}$ precipitates associated to $\mathrm{SiC}$ surface, including its ED patterns [ [ $\left.\begin{array}{lll}7 & \overline{1} & 1\end{array}\right]$. 

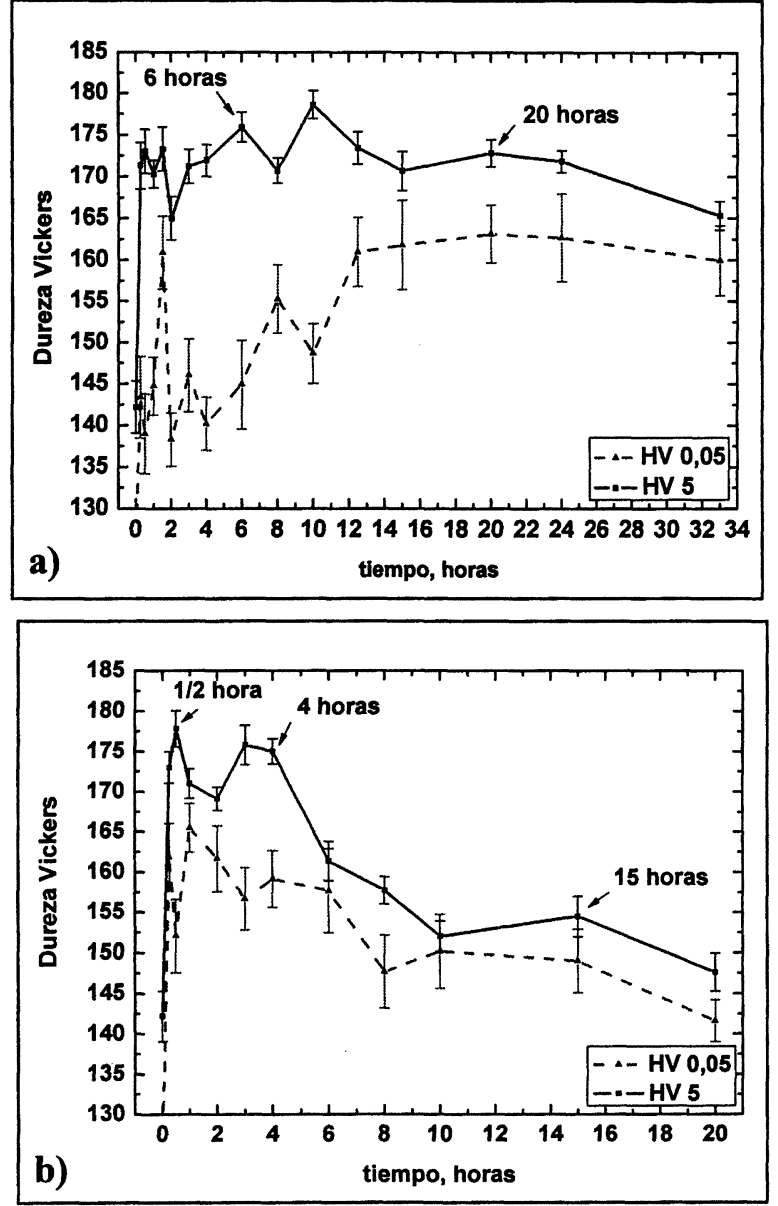

Figura 4. Perfiles de endurecimiento realizados a) a $170^{\circ} \mathrm{C}$ y b) a $190^{\circ} \mathrm{C}$.

Figure 4. a) Age hardening curves of the AA2009/SiC/15p composite aged at $170^{\circ} \mathrm{C}$. and b) aged at $190^{\circ} \mathrm{C}$.

ciertas subidas y bajadas en la dureza Vickers. Según aumenta el tiempo de tratamiento se observa un aumento de la dureza, más o menos rápido, dependiendo de las condiciones utilizadas, hasta alcanzar un máximo a partir del cual la dureza vuelve a descender.

Comparando las curvas de dureza, se puede decir que la influencia de la temperatura sobre el endurecimiento del material afecta sobre todo a la velocidad a la que se produce la precipitación. El incremento de la temperatura de envejecimiento acelera dicho proceso, alcanzándose los máximos de endurecimiento después de 0,5 y $4 \mathrm{~h}$ de tratamiento, cuando el tratamiento se realiza a $190^{\circ} \mathrm{C}$, mientras que, a $170^{\circ} \mathrm{C}$, dichos picos se sitúan a 6 y $20 \mathrm{~h}$, respectivamente.

El efecto observado respecto a los valores de dureza en dichos picos es el esperado, comprobándose como a mayor temperatura de maduración los valores de dureza alcanzados en estos máximos dis-

\section{2}

(c) Consejo Superior de Investigaciones Científicas

Licencia Creative Commons 3.0 España (by-nc) minuyen. Este efecto, que se aprecia más claramente en los valores de microdureza, genera una inversión en los valores relativos de endurecimiento en los dos picos, es decir, a $170^{\circ} \mathrm{C}$ el primer pico de dureza es mayor que el segundo y, sin embargo, a $190^{\circ} \mathrm{C}$ ocurre lo contrario.

A partir de los resultados obtenidos por calorimetría diferencial de barrido (DSC) se pueden explicar estas diferencias (Fig. 5). Se puede apreciar que los picos aparecen más desplazados hacia la derecha y más marcados si se aumenta la velocidad de calentamiento.

Los cambios exotérmicos están asociados a precipitaciones de fases y los endotérmicos a solubilizaciones. Por tanto, una gran respuesta exotérmica se correspondería con la precipitación que da lugar al pico de dureza medido. El primer pico exotérmico, que se relacionaría con la formación de zonas GPB, correspondería con el primer máximo de dureza de los tratamientos térmicos. El segundo parece estar formado por un doblete solapado y se correspondería con el segundo pico de dureza. Según bibliografía revisada sobre aleacio-

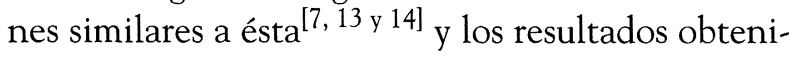
dos mediante TEM, el primer pico del doblete corresponde con la precipitación de la fase $S^{\prime}$ y el segundo con la de $\theta^{\prime}$. La tabla II resume los valores de temperaturas a las tienen lugar dichas transformaciones exotermicas.

Las micrografías de TEM del material tratado a 170 y $190^{\circ} \mathrm{C}$ en los dos picos de endurecimiento se muestran en las figuras 6 y 7 , respectivamente. $\mathrm{El}$ material envejecido durante $6 \mathrm{~h}$ a $170^{\circ} \mathrm{C}$ no

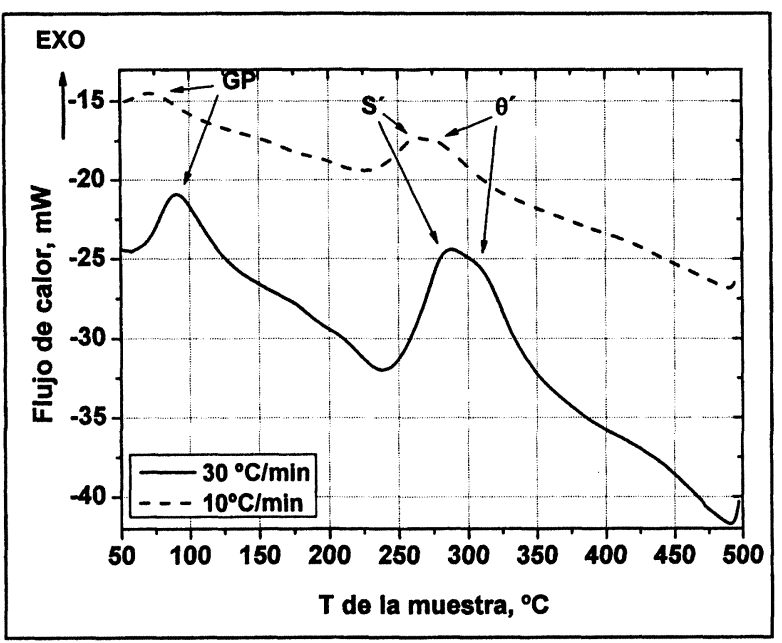

Figura 5. DSC del material solubilizado y templado, realizado a 30 (línea continua) y $10^{\circ} \mathrm{C} / \mathrm{min}$ (línea discontinua).

Figure 5. DSC thermograms of as-quenched material for 30 and $10^{\circ} \mathrm{C} / \mathrm{min}$ heating rates.

Rev. Metal. Madrid 41 (2005) 298-307

http://revistademetalurgia.revistas.csic.es 

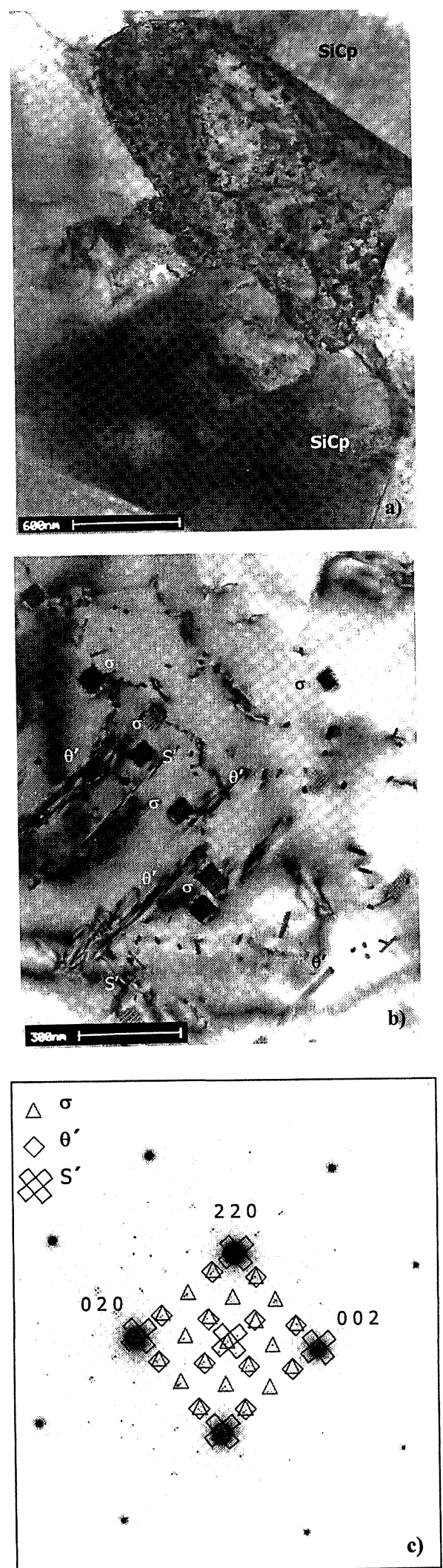

Figura 6. Imágenes de TEM del aspecto que ofrece la matriz del material tratado a $170{ }^{\circ} \mathrm{C}$, a) durante $\left.6 \mathrm{~h}, \mathrm{~b}\right) \mathrm{du}$ rante $20 \mathrm{~h}$, c) DE realizada sobre la matriz de éste en el eje de zona $\left[\begin{array}{lll}0 & 0 & 1\end{array}\right]$.

Figure 6. TEM images of the composite matrix aged at $170^{\circ} \mathrm{C}$, a) for $6 \mathrm{~h}, \mathrm{~b})$ for $20 \mathrm{~h}, \mathrm{cl}$ corresponding ED in [ $\left[\begin{array}{lll}0 & 0 & 1\end{array}\right]$ direction.
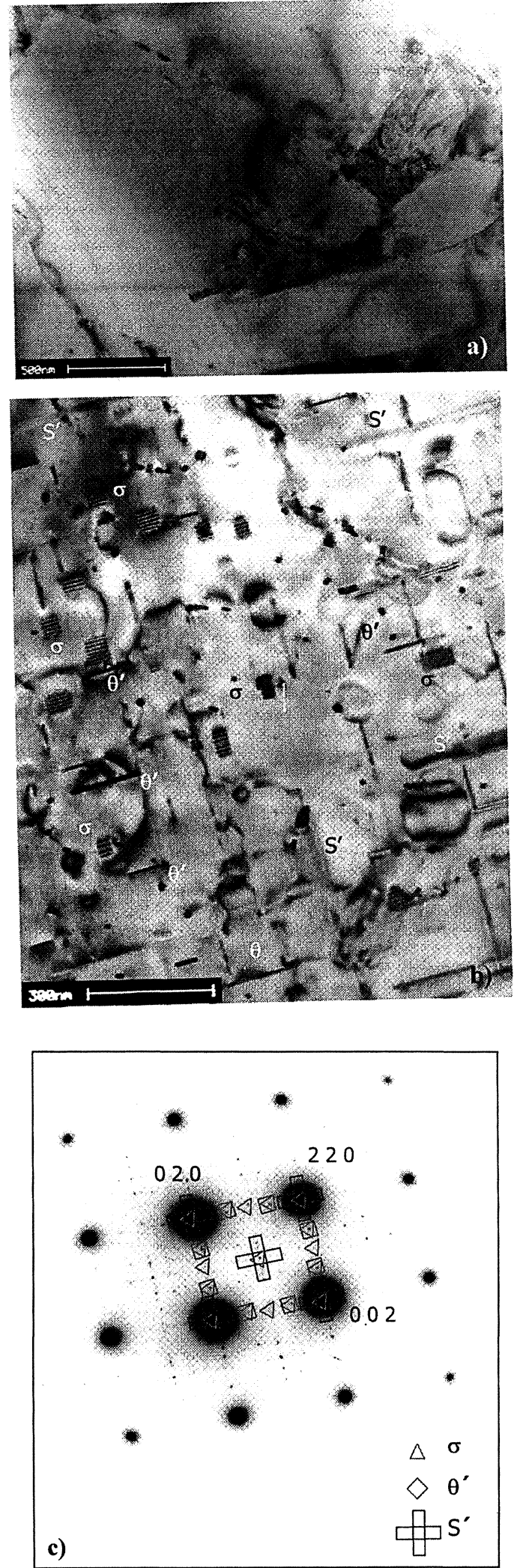

Figura 7. Imágenes de TEM del aspecto que ofrece la matriz del material tratado a $190^{\circ} \mathrm{C}$, a) durante $30 \mathrm{~min}$, b) durante $4 \mathrm{~h}, \mathrm{c}$ ) DE realizada sobre la matriz de éste en el eje de zona [ [ $\left.\begin{array}{lll}0 & 0 & 1\end{array}\right]$.

Figure 7. TEM images of the composite matrix aged at $190^{\circ} \mathrm{C}$, a) for $30 \mathrm{~min}$, b) for $4 \mathrm{~h}, \mathrm{c}$ ) corresponding ED in [ $\left[\begin{array}{lll}0 & 0 & 1\end{array}\right]$ direction. 
Relación entre los mecanismos de alimentación y el modo de solidificación en una aleación de aluminio 380 con distintos contenidos de hierro D.O. Tovio, A.C. GONZÁlez, G.W. Múgica y J.C. CuYÁs

Tabla Il. Temperatura máxima de las transformaciones exotérmicas

Table II. Peak temperature of the exothermic effects

\begin{tabular}{lcccc}
\hline \multirow{2}{*}{ Medio de temple } & Velocidad de calentamiento & \multicolumn{2}{c}{ Temperatura $\left({ }^{\circ} \mathrm{C}\right)$} \\
\cline { 3 - 5 } & & Formación de ZGP & Transformación IIIA & Transformación IIIB \\
\hline \multirow{2}{*}{$10^{\circ} \mathrm{C} / \mathrm{min}$} & 73 & 261 & 274 \\
Agua-hielo & & 72,5 & 263 & 276 \\
& $30{ }^{\circ} \mathrm{C} / \mathrm{min}$ & 89 & 275 & 295 \\
& & 90,7 & 288 & 307,5 \\
\hline
\end{tabular}

presenta fases precipitadas en la matriz, estando sus límites de grano e intercaras limpios de precipitados (fig. 6a). Sí se aprecia, por el contrario, una elevada densidad de dislocaciones asociada a la deformación de la matriz producida durante el temple. En la muestra madurada a $190^{\circ} \mathrm{C}$, en su primer pico de endurecimiento $(0,5 \mathrm{~h}$ ) (Fig. $7 \mathrm{a}$ ), se aprecia una disminución de la densidad de dislocaciones y una precipitación incipiente en límite de grano, lo que podría justificar los menores valores de dureza detectados en este primer pico, cuando el envejecimiento se realiza a mayor temperatura.

Respecto al estado de precipitación en las dos muestras observadas mediante TEM en el segundo pico de dureza (Figs. 6b y 7b), se aprecia una precipitación masiva de las dos fases endurecedores mayoritarias $\left(S^{\prime}\right.$ y $\left.\theta^{\prime}\right)$ : los $S^{\prime}$, en tono más claro, más desarrollados ya que empezarían a formarse antes, caracterizados por su forma de aguja, que se ve reflejada en los diagramas de difracción de electrones correspondientes en forma de "barritas" características de este precipitado (Figs. 6c y $7 \mathrm{c})$; los $\theta^{\prime}$, más oscuros y pequeños, que precipitan en forma de placas y se han identificado también por su reflexión en el diagrama de difracción de electrones correspondiente. Junto a estos precipitados, se ha identificado otra fase endurecedora minoritaria (fase $\sigma, \mathrm{Al}_{5} \mathrm{Cu}_{6} \mathrm{Mg}_{2}$ ), más abundante en el material madurado a $170^{\circ} \mathrm{C}$ durante $20 \mathrm{~h}, \mathrm{y}$ que tendría cierta responsabilidad en el mayor nivel de endurecimiento alcanzado en estas condiciones.

\subsection{Ensayos de tracción}

Los ensayos de tracción se realizaron sobre muestras tratadas, en condiciones de subenvejecimiento (primer pico de dureza), envejecimiento (segundo pico) y sobreenvejecimiento (Fig. 8). Se observa una mejora sustancial de la resistencia a tracción en todos los materiales tratados térmicamente. La
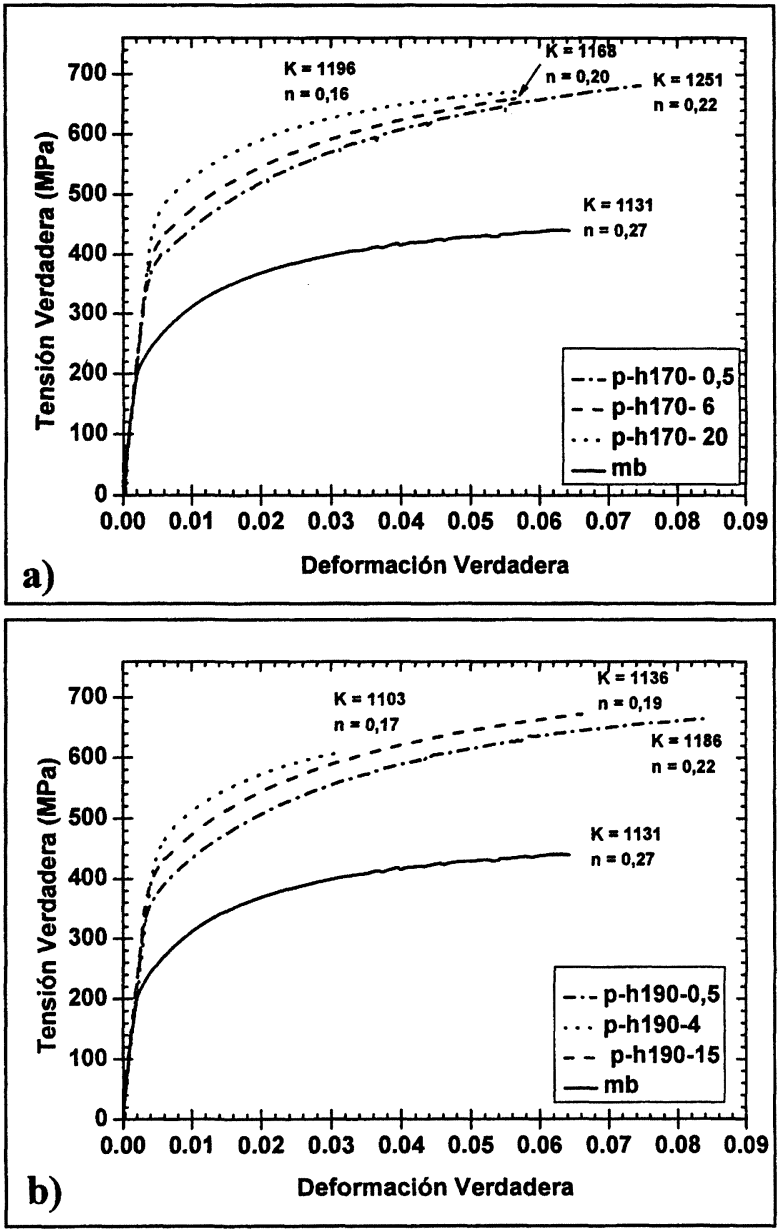

Figura 8. Curvas tensión-deformación del material de partida y tratado, a) a $170{ }^{\circ} \mathrm{C}$ y b) a $190^{\circ} \mathrm{C}$.

Figure 8. True stress-true strain curves of as-received and peak aged specimens. a) aged at $170{ }^{\circ} \mathrm{C}$. b) aged at $190^{\circ} \mathrm{C}$.

mayor ductilidad se alcanza en la condición de subenvejecimiento aunque, a costa de una reducción del límite elástico. En la condición envejecimiento aumenta el límite elástico pero disminuye la ductilidad. La tabla III resume los valores alcanzados para estas propiedades en las diferentes condiciones de envejecimiento empleadas. 
Tabla III. Propiedades mecánicas de tracción del material en estado de recepción y tratado

Table III. Average tensile properties of as-received and peak aged specimens

\begin{tabular}{llll}
\hline PROBETA & $\sigma_{0,2}(\mathrm{MPa})$ & $\sigma_{\mathrm{ts}}(\mathrm{MPa})$ & $\mathrm{A}(\%)$ \\
\hline p-MB & $245 \pm 6$ & $468 \pm 6$ & $6,4 \pm 0,6$ \\
p-H170-0,5 & $402 \pm 3$ & $681 \pm 3$ & $7,6 \pm 0,3$ \\
p-H170-6 & $435 \pm 4$ & $655 \pm 4$ & $5,6 \pm 0,1$ \\
p-H170-20 & $499 \pm 3$ & $667 \pm 3$ & $5,8 \pm 0,1$ \\
p-H190-0,5 & $383 \pm 5$ & $668 \pm 4$ & $8,2 \pm 0,3$ \\
p-H190-4 & $474 \pm 4$ & $612 \pm 3$ & $3,7 \pm 0,5$ \\
p-H190-15 & $428 \pm 12$ & $634 \pm 37$ & $6,2 \pm 0,5$ \\
\hline
\end{tabular}

A partir de las curvas tensión verdadera-deformación verdadera de la figura 8 , se pudo comprobar la existencia de una dependencia exponencial entre ambas propiedades, para valores de tensión superiores al límite elástico:

$$
\sigma_{\mathrm{t}}=K \varepsilon_{t}^{n}
$$

calculándose los correspondientes valores del coeficiente de resistencia $(K)$ y del exponente de endurecimiento por deformación (n), que se representan en la figura 8. Como puede comprobarse, el coeficiente de endurecimiento por deformación alcanza los valores mínimos (aproximadamente, $0,16)$ para los tiempos de envejecimiento a los que se alcanzan los picos de máximo endurecimiento del material (4 y $20 \mathrm{~h}$, para temperaturas de envejecimiento de 190 y $170^{\circ} \mathrm{C}$, respectivamente).

La rotura de los materiales ensayados se produjo por nucleación, crecimiento y coalescencia de huecos (Figs. 9 y 10). El mecanismo de rotura dominante en el material en estado de recepción fue la descohesión de la intercara matriz/refuerzo y la rotura de intermetálicos de $\mathrm{Al}_{2} \mathrm{Cu}$ de gran tamaño (Fig. 9b). El refuerzo cerámico no se rompió durante el ensayo de tracción, debido a su pequeño tamaño. La nucleación de huecos en la intercara predominó en las zonas formadas por agrupaciones de partículas, debido al aumento de la tensión hidrostática en la vecindad de los refuerzos.

Los intermetálicos de gran tamaño se disolvieron durante el tratamiento térmico y, por tanto, el mecanismo de rotura dominante fue la nucleación de huecos por descohesión en la intercara matriz/refuerzo (Figs. 10a y 10b). Puede observarse una población de huecos de tamaño inferior al encontrado en el material de recepción, que pudie-
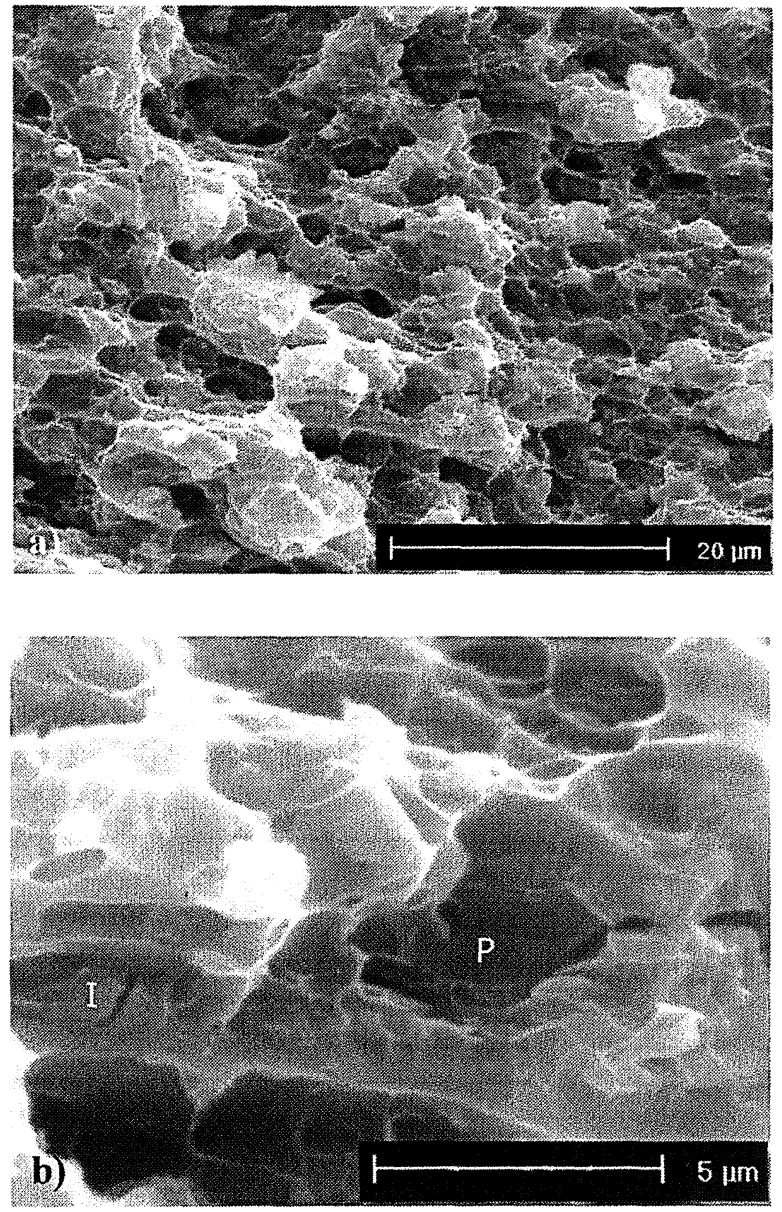

Figura 9. a) Fractografía del material en estado de recepción. b) Rotura de intermetálicos $\mathrm{Al}_{2} \mathrm{Cu}$ (marcados con I) y descohesión $\mathrm{Al} / \mathrm{SiCp}$ (marcada con P).

Figure 9. a) SEM image of fracture surface of as-received material. b) $\mathrm{Al}_{2} \mathrm{Cu}$ precipitate fracture (marked I) and particle/matrix decohesion (marked P).

ron formarse por los estados de tensión que generaron los precipitados endurecedores al anclar dislocaciones durante el ensayo de tracción.

La superficie pulida paralela a la dirección de carga mostró, no obstante, la existencia de algunos intermetálicos que no se disolvieron durante el tratamiento térmico (Fig. 11a). Se trata de fases intermetálicas que contienen hierro en su composición (Fig. 11b). Aunque estas aleaciones tienen un contenido muy reducido en estos elementos, éstos se combinan formando compuestos insolubles. Sin embargo, no se ha observado que afectaran al comportamiento mecánico del material.

\section{CONCLUSIONES}

- El tratamiento de envejecimiento artificial muestra dos picos de dureza, el primero de ellos 

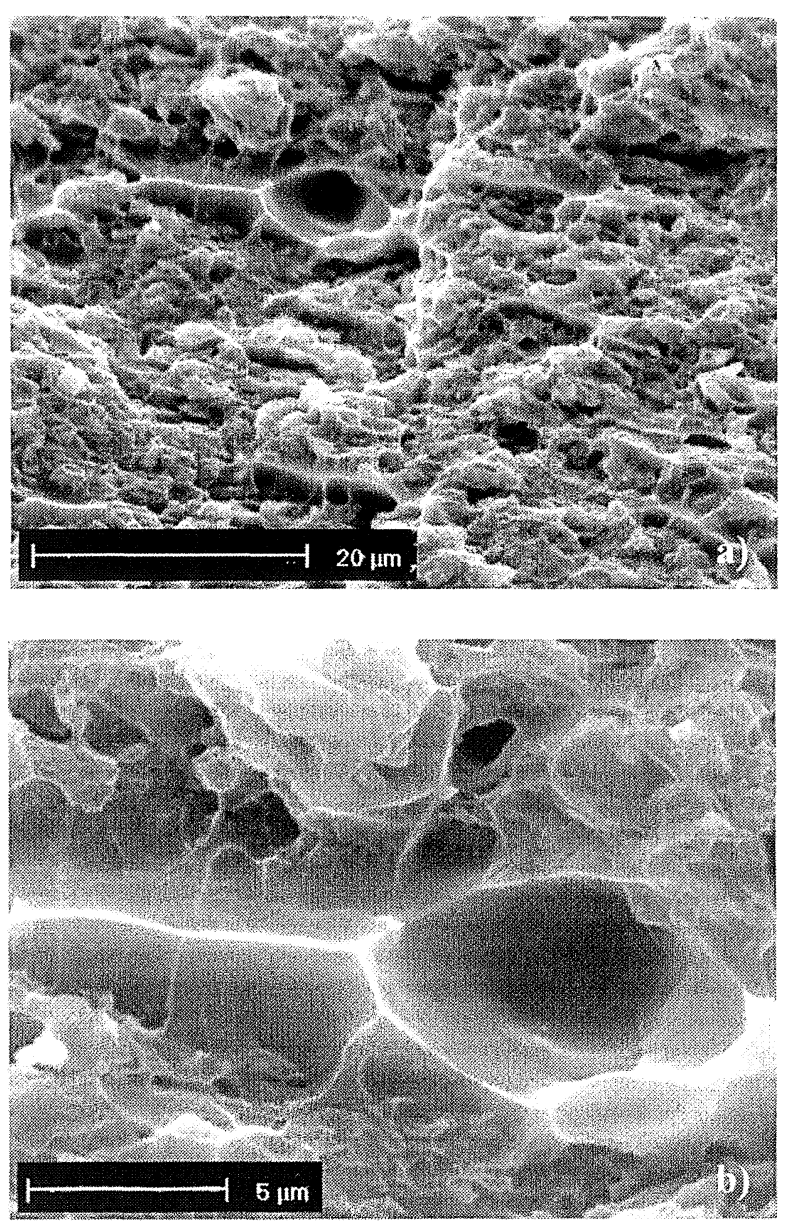

Figura 10. a) Fractografía del material tratado $4 \mathrm{~h}$ a 190 ${ }^{\circ} \mathrm{C}$. b) Hueco formado por descohesión de la intercara $\mathrm{Al} / \mathrm{SiCp}$.

Figure 10. a) SEM image of fracture surface of a specimen aged at $190{ }^{\circ} \mathrm{C}$ during $4 \mathrm{~h}$. b) Void formed by matrix decohesion around a SiC particle.

corresponde a la formación de zonas GP y el segundo a la precipitación de las fases endurecedores, $S^{\prime}$ y $\theta^{\prime}$.

- Mediante DSC se observan, también, dos transformaciones exotérmicas, la primera asociada a la formación de zonas GP y la segunda, constituida por un doblete, a la formación de $S^{\prime}$ y $\theta^{\prime}$. Las observaciones de TEM corroboraron que el primer pico del doblete corresponde a la formación de $S^{\prime}$ y el segundo a la de $\theta^{\prime}$, basándose en el mayor tamaño de los primeros.

- El tratamiento térmico mejora en todos los casos la resistencia a tracción y el límite elástico. El valor más elevado de ductilidad corresponde con el estado de subenvejecimiento.

- La rotura del material en estado de recepción se produjo por nucleación, crecimiento y coalescencia de huecos. La rotura se inició por descohesión de la intercara matriz/refuerzo y por la
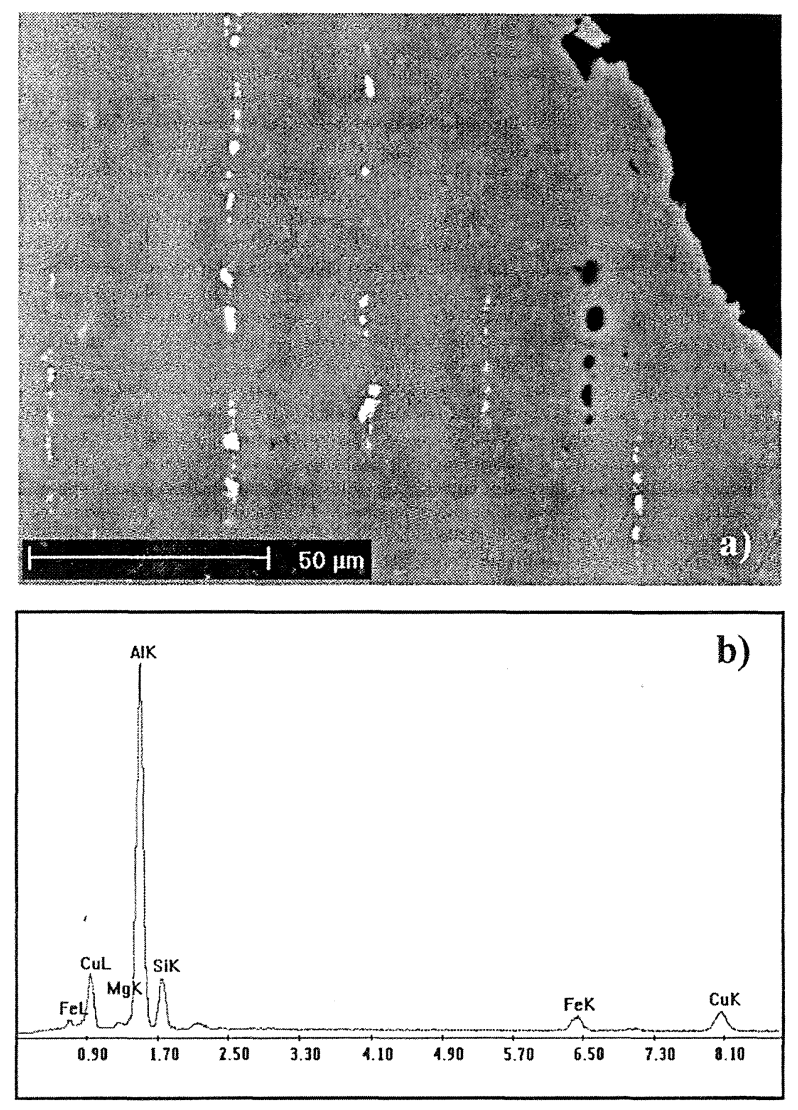

Figura 11. a) Superficie longitudinal pulida, presencia de intermetálicos insolubles con Fe y Si (brillantes). b) Análisis EDX realizado sobre uno de estos precipitados.

Figure 11. a) Longitudinal cross section showing insoluble intermetallics riched in Fe and Cu. b) EDX microanalysis of them.

rotura de intermetálicos $\mathrm{Al}_{2} \mathrm{Cu}$ de gran tamaño.

- La rotura de los materiales tratados está controlada sólo por la descohesión de la intercara matriz/refuerzo, debido a la solubilización de intermetálicos durante el tratamiento térmico.

\section{AGRADECIMIENTOS}

Los autores desean agradecer al Ministerio de Educación Ciencia por la ayuda económica recibida para la realización de la presente investigación (Proyecto MAT2004-06018).

\section{REFERENCIAS}

[1] D.J. LloYd, Inter. Mat. Rev. 39 (1994) 1-23.

[2] T.W. Clyne y D.S. Withers, An introduction to metal matrix composites, Cambridge University Press, 1993.

[3] N.M. Piris, J.N.M. Badía, J.M. Antoranz y P. Tarín, Rev. Metal. Madrid 40 (2004) 288-293. 
[4] A. Monsalve y R. Morales, Rev. Metal. Madrid 40 (2004) 431-435.

[5] R.J. ARSENAult Y R.M. FISHER, Scr. Metall. 17 (1983) 67-71.

[6] S.R. Nutt y A. NeEdleman. Scr. Metall. 21 (1987) 705. 710 .

[7] C. Badini, F. Marino y E. Verné, Mater. Sci. Eng. A191 (1995) 185-191.

[8] J. LloRCA, Void Formation in Metal Matrix Composites. Comprehensive Composite Materials, Vol. 3, (T. W. Clyne, Ed.), Pergamon, Amsterdam, 2000, pp. 91-115.

[9] B. DeRby, P.M. MuMMERY y C.W. LAWRENCE, Intrinsic and extrinsic fracture mechanisms in inorganic composite systems,
(Eds. J.J.Lewandowski and H.H. Warren), T.M.S., 1995, pp. 7-13.

[10] A.P. SanNino y H.J. Rack, J. Mater. Sci. 30 (1995) 4.316-4.322.

[11] NORMA UNE-EN 10002, 2002, 27-29.

[12] A. Ureña, P. Rodrigo, L. Gil, M.D. Escalera Y J.L. BALDONEDO, J. Mater. Sci. 36 (2001) 419-428.

[13] I. DutTA, C.P. Harper Y G. DutTA, Metall. Mater. Trans. A 25A (1994) 1.591-1.602.

[14] I.N.A. Oguocha y S. Yannacopoulos, J. Mater. Sci. 34 (1999) 3.335-3.340. 


\section{FE DE ERRATAS}

Págs. 299-307

En la cabecera de cada una de las páginas, dice:

"Relación entre los mecanismos de alimentación y el modo de solidificación en una aleación de aluminio 380 con distintos contenidos de hierro

D.O. TOVIO, A.C. GONZÁLEZ, G.W. MÚGICA Y J.C. CUYAS" y debe decir:

"Influencia del estado de envejecimiento en el comportamiento mecánico de una aleación de aluminio AA2009 reforzada con partículas de $\mathrm{SiC}$

R RODRIGO, R POZA, M.V. UTRILLA Y A. UREÑA". 